\title{
Evaluation of Meibomian Gland Dysfunction Among Ophthalmic Healthcare Workers
}

This article was published in the following Dove Press journal:

Clinical Ophthalmology

\author{
Alison YY Chan ${ }^{1,2, *}$ \\ Jasmine C Chuang ${ }^{1,2, *}$ \\ Victoria WY Wong ${ }^{1-3}$ \\ 'Hong Kong Eye Hospital, Kowloon, \\ Hong Kong; ${ }^{2}$ Department of \\ Ophthalmology and Visual Sciences, The \\ Chinese University of Hong Kong, \\ Hong Kong; ${ }^{3} \mathrm{HKU}$ Health System, Li Ka \\ Shing Faculty of Medicine, University of \\ Hong Kong, Hong Kong
}

*These authors contributed equally to this work
Correspondence: Victoria WY Wong HKU Health System, Li Ka Shing Faculty of Medicine, University of Hong Kong, 2I Sassoon Road, Pokfulam, Hong Kong

Email drvwong@hotmail.com
Purpose: To evaluate the prevalence of meibomian gland dysfunction (MGD) among ophthalmic healthcare workers.

Setting: A tertiary ophthalmic center.

Design: Prospective, observational study.

Methods: Forty-four volunteer ophthalmologists and ophthalmic nurses were recruited. Information including demographics, contact lens wear, history of refractive surgery and symptom score based on Standardized Patient Evaluation of Eye Dryness (SPEED) II Questionnaire for Dry Eye Disease/Ocular Surface Disease were recorded. Lipid layer thickness (LLT), meibomian glands dropout and dilation grades, and proportion of partial blinking were evaluated using an ocular surface interferometer with dynamic meibomian imaging (LipiView, Johnson \& Johnson). Based on the chance of MGD, meibomian gland dropout and dilation, selected subjects also underwent treatment with a thermal pulsation system (LipiFlow, Johnson \& Johnson) in one or both eyes.

Results: Eighty-eight eyes of 44 volunteers were evaluated during the study period. The mean LLT was $60.0 \mathrm{~nm}$. Twenty-seven $(61.4 \%)$ subjects had a $90 \%$ or high chance of MGD and their mean lower lid meibomian gland dropout and dilation grades were 1.2 and 1.7, respectively. Twenty-eight eyes of 16 volunteers received treatment with the thermal pulsation system. Following treatment, the mean LLT improved from $50.3 \mathrm{~nm}$ to $61.0 \mathrm{~nm}$ (Wilcoxon's signed rank test, $\mathrm{p}=0.001$ ).

Conclusion: Despite being more knowledgeable to MGD and more accessible to treatment, MGD is a highly prevalent condition among ophthalmic healthcare workers, with a $61.4 \%$ prevalence among the recruited subjects. This is similar to reported prevalence in Asian populations of up to $74.5 \%$. Targeted therapy based on dynamic meibomian imaging is effective in improving both objective and subjective measures of MGD.

Keywords: meibomian gland dysfunction, lipid layer thickness, dynamic meibomian imaging, thermal pulsation system

\section{Introduction}

The term meibomian gland dysfunction (MGD) was first coined by Korb and Henriquez in $1980 .{ }^{1}$ In 2011, the International Workshop on Meibomian Gland Dysfunction (IWMGD) defined MGD as a chronic, diffuse abnormality of the meibomian glands, commonly characterized by terminal duct obstruction and/or qualitative or quantitative changes in the glandular secretion. ${ }^{2}$ Terminal meibomian duct obstruction and dilatation lead to gland dropout, atrophy and reduced or altered lipid secretion. The subsequent impaired delivery of lipids to the ocular surface precipitates tear film instability and ultimately evaporative dry eye. MGD has been 
reported to account for $60 \%$ of all cases of dry eye disease and might be more prevalent in Asian populations, ${ }^{3}$ with a reported prevalence of up to $74.5 \% .^{3-6}$

Recent advancement in ocular surface interferometers with dynamic meibomian imaging such as Keratograph and LipiView ocular surface interferometers has enhanced the ability in diagnosing MGD. ${ }^{7,8}$ It provides objective measurements of tear film lipid layer thickness (LLT), as well as infrared images for evaluation of meibomian gland dropout and duct dilation. Finis et al reported a sensitivity and specificity for MGD diagnosis of $65.8 \%$ and $63.4 \%$ respectively when an LLT cutoff value of $\leq 75 \mathrm{~nm}$ was used, and a sensitivity and specificity of $47.9 \%$ and $90.2 \%$ respectively when an LLT cutoff value of $\leq 60 \mathrm{~nm}$ was used. ${ }^{9}$ While these values may not suffice for clinical use, the sensitivity and specificity may be enhanced by integrating LLT values with other parameters such as symptoms score, lid margin abnormality and meibography scores. ${ }^{10}$

As MGD is one of the most common ophthalmic conditions and is a major cause of dry eye disease, ophthalmic healthcare workers including ophthalmologists and ophthalmic nurses need to educate patients on measures to prevent and treat MGD on a daily basis. It would be of interest to evaluate the prevalence of MGD among ophthalmic healthcare workers. The use of ocular surface interferometer with dynamic meibomian imaging enables subjects and ophthalmic staff to directly visualize the MGD disease process, which can provide a basis for health education and behavior modification. The aims of this study were to evaluate the prevalence of MGD among ophthalmic healthcare workers in a tertiary eye center using the ocular surface interferometer with dynamic meibomian imaging and to assess how interventions like thermal pulsation system therapy in selected subjects can improve their dry eye symptoms.

\section{Methods}

\section{Setting and Subjects}

Volunteer ophthalmologists and ophthalmic nurses were recruited in the Hong Kong Eye Hospital in September 2019. They were excluded if they had any ocular history of trauma, red eye, infection or inflammation, foreign body or surgery within the preceding 3 months. Written informed consent was obtained from all subjects in the study. The study was performed in accordance with the Declaration of the Helsinki and was reviewed and approved by the Hong Kong Hospital Authority's Kowloon Central/ Kowloon East Cluster Research Ethics Committee.

\section{Evaluations}

Information including demographics, contact lens wear, history of refractive surgery, regular lid hygiene practice and lubricant use, and symptom score based on Standardized Patient Evaluation of Eye Dryness (SPEED) II Questionnaire for Dry Eye Disease/Ocular Surface Disease were collected through a questionnaire. Lipid layer thickness (LLT) and proportion of partial blinking were evaluated using an ocular surface interferometer with dynamic meibomian imaging (LipiView, Johnson \& Johnson Inc., New Brunswick, NJ). The LLT cutoff values for diagnosis of MGD measured using the LipiView interferometer were based on studies by Finis et al and Blackie et al. ${ }^{9,11}$ The risks of MGD were classified as 1) " $90 \%$ probability of MGD" is defined as LLT $<60 \mathrm{~nm}$ bilaterally; 2) "high risk" is defined as LLT $<60 \mathrm{~nm}$ in at least one eye; 3) "medium risk" is defined as LLT $60-75 \mathrm{~nm}$ in at least one eye; and 4) "low risk" as LLT $>75 \mathrm{~nm}$ in at least one eye. ${ }^{9}$ Eyes graded to have " $90 \%$ probability" and "high risk" of MGD were further evaluated for meibomian glands dropout and gland duct dilation using dynamic meibomian imaging. The meibomian glands dropout and duct dilations in the infrared images of both lower eyelids were graded by two observers using the MeiboGrade $_{\mathrm{TM}}$ pictorial reference guide developed by Blackie and Korb provided by the manufacturer (Johnson \& Johnson Inc., New Brunswick, NJ). Meibomian glands dropout was graded as 0 (no loss of Meibomian glands), grade 1 (dropout of $<1 / 3$ of total Meibomian glands), grade 2 (dropout of 1/3-2/3 of total Meibomian glands) and grade 3 (dropout of $>2 / 3$ total Meibomian glands), as proposed by Arita et al. ${ }^{12}$ Gland duct dilation was graded as 0 (no gland duct dilation), grade 1 (mild duct dilation), grade 2 (moderate dilation) and grade 3 (severe dilation). Subjects with " $90 \%$ probability" or "high risk" of MGD based on LLT, together with gland duct dilation grades of 2-3 and gland dropout severity grades of $0-1$, were selected for thermal pulsation system treatment (LipiFlow, Johnson \& Johnson Inc., New Brunswick, NJ). During the thermal pulsation system treatment session, subjects were also taught to blink more fully in order to reduce partial blinking.

\section{Statistical Analyses}

The LLT and SPEED II symptom scores of the entire group, pre-treatment and post-treatment were recorded and compared. Continuous variables were compared 
with the Wilcoxon's signed-rank test and categorical variables with the Fisher's exact test. Spearman correlation test was used to determine the correlation between subjective symptoms of dry eye disease and objective signs of MGD. All tests were computed using StatPlus: mac Pro v.5.9.5 (AnalystSoft Inc, Walnut, CA). A twotailed p-value of $<0.05$ was considered as statistically significant.

\section{Results}

Eighty-eight eyes of 44 volunteers (21 ophthalmologists and 23 ophthalmic nurses) were recruited, among which $41(93.2 \%)$ were female and three $(6.8 \%)$ were male. The 31-40 years age group constituted the largest proportion of subjects with $18(40.9 \%)$ subjects (Table 1). At baseline evaluation, the mean LLT was $60.0 \mathrm{~nm}$ (range, $46.2 \mathrm{~nm}$ to $73.3 \mathrm{~nm})$. Twenty-seven (61.4\%) subjects were classified as having $90 \%$ or high risk of MGD, seven $(15.9 \%)$ as medium risk and ten $(22.7 \%$ ) as low risk (Table 2). Partial blinking occurring with more than $40 \%$ of blinks were present in $53(60.3 \%)$ eyes. Among the 27 subjects with $90 \%$ or high chance of MGD, the mean and median lower lid meibomian gland dropout grades were 1.2 and 1.0 respectively, and the mean and median lower lid meibomian gland dilation grades were 1.7 and 1.5 , respectively.

Table I Demographics of the 44 Subjects in the Study

\begin{tabular}{|l|c|c|}
\hline \multicolumn{2}{|l|}{ Characteristics } & Number (\%) \\
\hline Age group (years) & $2 I-30$ & $9(20.5)$ \\
& $3 I-40$ & $18(40.9)$ \\
& $4 I-50$ & $9(20.5)$ \\
& $5 I-60$ & $8(16.5)$ \\
\hline & Male & $3(6.8)$ \\
& Female & $4 I(93.2)$ \\
\hline Contact lens wear & Yes & $20(45.5)$ \\
& No & $24(55.5)$ \\
\hline History of refractive surgery & Yes & $3(6.8)$ \\
\hline History of blepharitis & No & $4 I(93.2)$ \\
\hline Regular lid hygiene & Yes & $5(11.4)$ \\
& No & $39(88.6)$ \\
\hline Topical lubricant use & Yes & $10(22.7)$ \\
& No & $34(77.3)$ \\
\hline Subjective symptom of dry eye & Yes & $30(68.2)$ \\
disease & No & $14(32.8)$ \\
\hline
\end{tabular}

Table 2 Distribution of 44 Subjects by Risk of Meibomian Gland Dysfunction

\begin{tabular}{|l|c|c|}
\hline Risk of MGD & $\begin{array}{c}\text { Number of } \\
\text { Subjects } \\
\text { (n=44) }\end{array}$ & $\begin{array}{c}\text { Percentage } \\
\text { (\%) }\end{array}$ \\
\hline $\begin{array}{l}\text { 90\% chance (LLT <60nm } \\
\text { bilaterally) }\end{array}$ & 20 & 45.5 \\
\hline $\begin{array}{l}\text { High chance (LLT <60nm in at } \\
\text { least I eye) }\end{array}$ & 7 & 15.9 \\
\hline $\begin{array}{l}\text { Medium chance (LLT 60-75nm in at } \\
\text { least I eye) }\end{array}$ & 7 & 15.9 \\
\hline $\begin{array}{l}\text { Low chance (LLT >75nm in at } \\
\text { least I eye) }\end{array}$ & 10 & 22.7 \\
\hline
\end{tabular}

Abbreviations: MGD, meibomian gland dysfunction; LLT, lipid layer thickness; nm, nanometer.

The overall mean \pm standard deviation (SD) baseline SPEED II symptom score was $10.4 \pm 6.0$ (range, 2 to 28). Comparing ophthalmologists versus ophthalmic nurses, there was no significant difference in the mean subjective SPEED II symptom scores (mean 9.6 vs 11.2 , respectively, $\mathrm{P}=0.20$, Mann-Whitney $U$-test) and objective LLT measurements (12 ophthalmologists vs 14 nurses were graded as $90 \%$ or high change of MGD, $\mathrm{P}=0.80$, chi-squared test). In terms of age, no significant association was found between the age groups and SPEED scores $(\mathrm{P}=0.40$, Kruskal-Wallis ANOVA test $)$ and the risk of $\mathrm{MGD}(\mathrm{P}=0.63$, chi-squared test). There was also no significant correlation between the subjects' subjective SPEED II symptom score and objective LLT measurement (Spearman's rho=-0.013, $\mathrm{p}=0.93)$. Although 30 (68.2\%) subjects had subjective symptoms of dry eye disease based on SPEED II symptom scores of 7 or higher, only ten $(22.7 \%)$ practiced regular lid hygiene prior to the study.

Sixteen volunteers (30 eyes) received treatment with the thermal pulsation system in one or both eyes. The baseline mean lower lid meibomian gland dropout and duct dilation grades were 1.0 and 2.1, respectively. At 4 weeks after treatment, the mean LLT of the treated subjects improved significantly from $50.3 \mathrm{~nm}$ to $61.0 \mathrm{~nm}$ (Wilcoxon's signed rank test, $\mathrm{p}=0.001$ ). The proportion of treated eyes which exhibited partial blinking of more than $40 \%$ of blinks also reduced significantly from $60.7 \%$ to $17.9 \%$ (Fisher's exact test, $\mathrm{p}=0.002$ ). Moreover, there was a statistically significant improvement in SPEED II questionnaire scores from a mean $\pm \mathrm{SD}$ of $12.9 \pm 1.30$ to $8.69 \pm 0.74$ (Wilcoxon's signed rank test, $\mathrm{p}=0.006$ ) at 4 weeks after thermal pulsation system treatment (Table 3). 
Table 3 Comparison of Pre- and Post-Treatment Parameters

\begin{tabular}{|l|c|c|c|}
\hline Parameter & $\begin{array}{c}\text { Pre-Treatment } \\
(\mathbf{n}=16)\end{array}$ & $\begin{array}{c}\text { Post-Treatment } \\
(\mathbf{n}=16)\end{array}$ & P-value \\
\hline Mean LLT (nm) & 50.3 & 61.0 & $0.001^{*}$ \\
\hline $\begin{array}{l}\text { Partial blinking } \\
>40 \%(\%)\end{array}$ & 60.7 & 17.9 & $0.002 \#$ \\
\hline $\begin{array}{l}\text { SPEED II } \\
\text { questionnaire } \\
\text { score }\end{array}$ & $12.94 \pm 1.30$ & $8.69 \pm 0.74$ & $0.006^{*}$ \\
\hline
\end{tabular}

Notes: *Wilcoxon's signed rank test; \#Fisher exact test.

Abbreviations: LLT, lipid layer thickness; nm, nanometer; $n$, number; SPEED, Standardized Patient Evaluation of Eye Dryness.

\section{Discussion}

In our study, $68.2 \%$ of ophthalmic healthcare workers had subjective symptoms of dry eye disease and $61.4 \%$ had signs of MGD, which were similar to reported prevalence rates of dry eye in population-based studies in Asia, in which prevalence of up to $74.5 \%$ has been reported. ${ }^{4-7}$ The age of our subjects ranged from 21 to 60 years, with a male-to-female ratio of 3:41. Several studies have described an association between increasing age and the prevalence of meibomian gland changes such as abnormal lid margin anatomy, meibum hyposecretion and meibomian gland dropout. ${ }^{13,14}$ Therefore, it can be expected that older individuals will have a higher prevalence rate of MGD. ${ }^{15}$ Due to the limited age range and small number of male subjects in our study, we were unable to compare the prevalence of MGD between age and gender.

Since MGD is a very common eye disorder, an objective diagnostic tool is extremely helpful for ophthalmic healthcare workers to allow patients to visualize the severity of MGD and to individualize the treatment strategy. In our study, we utilized the LipiView ocular surface interferometer with dynamic meibomian imaging as it has been shown to be useful in evaluating meibomian gland changes in MGD. ${ }^{7}$ The system also served as an education tool as it enabled both patients and healthcare workers to directly visualize the MGD disease process and compare the changes before and after treatment. For example, we found a significant reduction in subjects with partial blinking from $60.7 \%$ to $17.9 \%$ before and after thermal pulsation system treatment. This demonstrated that patients with a high partial blinking rate could be taught to blink more fully in order to improve the distribution of the tear film. Moreover, only $22.7 \%$ of our subjects performed regular lid hygiene, even when $68.2 \%$ considered themselves symptomatic. Prior to the use of dynamic meibomian imaging, our subjects might not have been aware that they have MGD. In this sense, the dynamic meibomian imaging enabled a deeper understanding of the disease process not just for our patients but also for ophthalmic staff, which may enhance the communication, diagnosis and management of patients with this common disorder. These findings are useful for both ophthalmologists and ophthalmic nurses as they will be providing MGD evaluation and treatment services to patients in our hospital with the ocular surface interferometer with dynamic meibomian imaging and thermal pulsation system.

Our study showed no significant correlation between subjective SPEED II symptom score and objective LLT measurement. This demonstrated that dry eye symptoms generally do not correlate well with signs of dry eye disease severity including MGD and is consistent with findings by Nichols et al. ${ }^{16}$ Since it would be very difficult for patients and healthcare workers to rely solely on subjective symptoms in managing MGD, the objective tools such as dynamic meibomian imaging system can therefore facilitate the development of treatment algorithms tailored for each patient's individual needs. ${ }^{17}$ In addition, MGD is known to have a biphasic disease course, whereby a preclinical symptom-free stage is followed by a symptomatic stage. ${ }^{10}$ Subjects with meibomian gland dilatation but who are asymptomatic can therefore be diagnosed at an earlier, more treatable stage prior to the development of irreversible gland atrophy and offered treatment more promptly.

The management of MGD is multifactorial, including lid hygiene, eyelid massage devices, and pharmacological agents such as lubricants, antibiotics, cyclosporine, topical corticosteroids, omega-3-fatty acids, and mucin analogues. ${ }^{18}$ In general, lid warming and mechanical massage are recommended in the initial stages, while the use of medications is reserved for non-responders and more severe disease. The IWMGD released a comprehensive review of management options for MGD in 2011, ${ }^{18}$ which also included several relatively new treatment modalities such as electronic eyelid-heating devices, ${ }^{19,20}$ thermal pulsation system, ${ }^{21,22}$ intense pulsed light therapy (IPL), ${ }^{23}$ intranasal tear neurostimulation, ${ }^{24}$ and meibomian gland duct probing. ${ }^{25}$ Amid the abundance of these treatment modalities, decisions concerning MGD management should be individualized based on symptoms and disease severity, ease of use, side effect profile, treatment response and costs. 
Eyelid-warming devices are based on the principle that MGD results in an altered meibum composition with a higher melting point, which requires higher gland temperature to liquefy and prevent obstruction. ${ }^{19,20}$ Increased eyelid temperature has been linked to reduced meibomian lipid viscosity and increased lipid levels on the lid margin. ${ }^{26}$ There are several eyelid-warming devices available commercially which provide heat at safe temperatures. The thermal pulsation system is a device used to warm and mechanically massage the eyelids and has been shown to be effective in reducing symptoms and improving meibomian gland function in patients with MGD..$^{21,22,27}$ Meibomian glands that are dilated but not yet atrophic are believed to be most responsive to treatment. ${ }^{28}$ In our study, subjects with an overall high risk of MGD alongside with high gland dilatation and low gland dropout grades were selected to receive thermal pulsation system treatment with LipiFlow. On the other hand, subjects at equally high risk of MGD but with high gland dropout rates might not benefit from thermal pulsation system treatment and were therefore excluded from treatment. Consistent with previous studies, ${ }^{29-32}$ our study demonstrated a statistically significant improvement in the mean LLT as well as mean SPEED II symptom scores of treated subjects. Nevertheless, in the public healthcare setting, the widespread use of thermal pulsation systems may be limited by its cost implications and need for repeated treatments. Instead, the thermal pulsation system may be more suitably employed as a one-off tool for patients to experience symptoms improvement, which in turn motivates compliance with daily lid hygiene and warm compresses.

There were several limitations in this study. The method by which subjects were recruited could have resulted in volunteer bias. The age and gender distribution of our subjects was not uniform and might have underestimated the prevalence rate of MGD. In addition, other objective parameters of dry eye such as tear film breakup time, Schirmer's test, and corneal fluorescein stain score were not performed at baseline. Moreover, we did not perform dynamic meibomian imaging in subjects with moderate or low risk of MGD and after the thermal pulsation system treatment. This is because we aimed to minimize the invasiveness of the investigations among the volunteers.

In conclusion, MGD is a common condition even among ophthalmic healthcare workers. In this study, we found the prevalence rate of MGD in ophthalmic staff to be similar to reported rates in Asian population-based studies. Furthermore, as blinking and its effect on the tear film is a dynamic process, real-time visualization of the tear film together with meibomian gland imaging would allow greater understanding of the MGD disease process and bring a paradigm shift to our current static investigations for MGD and dry eye diseases. This will in turn bring a clearer perspective to both patients and ophthalmic staff, allowing more targeted individualized management for patients with this common ocular surface disorder.

\section{Acknowledgments}

Alison Y.Y. Chan and Jasmine C. Chuang are co-first authors for this study. This study received material support from Hong Kong Johnson \& Johnson Inc. This study was presented in part as an oral presentation at the Hong Kong Ophthalmological Symposium in December 2019 in Hong Kong.

\section{Disclosure}

The authors report no conflicts of interest in this work.

\section{References}

1. Korb DR, Henriquez AS. Meibomian gland dysfunction and contact lens intolerance. J Am Optom Assoc. 1980;51(3):243-251.

2. Nelson JD, Shimazaki J, Benitez-del-Castillo JM, et al. The international workshop on meibomian gland dysfunction: report of the definition and classification subcommittee. Invest Ophthalmol Vis Sci. 2011;52(4):1930-1937. doi:10.1167/iovs.10-6997b

3. Lekhanont K, Rojanaporn D, Chuck RS, Vongthongsri A. Prevalence of dry eye in Bangkok, Thailand. Cornea. 2006;25(10):1162-1167. doi:10.1097/01.ico.0000244875.92879.1a

4. Schaumberg DA, Nichols JJ, Papas EB, Tong L, Uchino M, Nichols KK. The international workshop on meibomian gland dysfunction: report of the subcommittee on the epidemiology of, and associated risk factors for, MGD. Invest Ophthalmol Vis Sci. 2011;52 (4):1994-2005. doi:10.1167/iovs.10-6997e

5. Siak JJ, Tong L, Wong WL, et al. Prevalence and risk factors of meibomian gland dysfunction: the Singapore Malay eye study. Cornea. 2012;31:1223-1228. doi:10.1097/ICO.0b013e31823f0977

6. Amano S, Inoue K. Clinic-based study on meibomian gland dysfunction in Japan. Invest Ophthalmol Vis Sci. 2017;58:1283-1287. doi:10.1167/iovs.16-21374

7. Cochener B, Cassan A, Omiel L. Prevalence of meibomian gland dysfunction at the time of cataract surgery. J Cataract Refract Surg. 2018;44(2):144-148. doi:10.1016/j.jcrs.2017.10.050

8. Zang S, Cui Y, Cui Y, Fei W. Meibomian gland dropout in Sjögren's syndrome and non-Sjögren's dry eye patients. Eye (Lond). 2018;32 (11):1681-1687. doi:10.1038/s41433-018-0149-5

9. Finis D, Pischel N, Schrader S, Geerling G. Evaluation of lipid layer thickness measurement of the tear film as a diagnostic tool for Meibomian gland dysfunction. Cornea. 2013;32(12):1549-1553. doi:10.1097/ICO.0b013e3182a7f3e1 
10. Tomlinson A, Bron AJ, Korb DR, et al. The international workshop on meibomian gland dysfunction: report of the diagnosis subcommittee. Invest Ophthalmol Vis Sci. 2011;52(4):2006-2049. doi:10.1167/iovs.10-6997f

11. Blackie CA, Solomon JD, Scaffidi RC, et al. The relationship between dry eye symptoms and lipid layer thickness. Cornea. 2009;28(7):789-794. doi:10.1097/ICO.0b013e318191b870

12. Arita $\mathrm{R}$, Itoh $\mathrm{K}$, Inoue $\mathrm{K}$, Amano $\mathrm{S}$. Noncontact infrared meibography to document age-related changes of the meibomian glands in a normal population. Ophthalmology. 2008;115(5):911-915. doi:10.1016/j.ophtha.2007.06.031

13. Den S, Shimizu K, Ikeda T, Tsubota K, Shimmura S, Shimazaki J. Association between meibomian gland changes and aging, sex, or tear function. Cornea. 2006;25(6):651-655. doi:10.1097/01. ico.0000227889.11500.6f

14. Hykin PG, Bron AJ. Age-related morphological changes in lid margin and meibomian gland anatomy. Cornea. 1992;11(4):334-342. doi:10.1097/00003226-199207000-00012

15. Chatterjee S, Agrawal D, Sharma A. Meibomian gland dysfunction in a hospital-based population in central India. Cornea. 2020;39 (5):634-639. doi:10.1097/ICO.0000000000002217

16. Nichols KK, Nichols JJ, Mitchell GL. The lack of association between signs and symptoms in patients with dry eye disease. Cornea. 2004;23(8):762-770. doi:10.1097/01.ico.0000133997. $07144.9 \mathrm{e}$

17. Thulasi P, Djalilian AR. Update in current diagnostics and therapeutics of dry eye disease. Ophthalmology. 2017;124(11S):S27-S33. doi:10.1016/j.ophtha.2017.07.022

18. Nichols KK, Foulks GN, Bron AJ, et al. The international workshop on meibomian gland dysfunction: executive summary. Invest Ophthalmol Vis Sci. 2011;52(4):1922-1929. doi:10.1167/iovs.10$6997 \mathrm{a}$

19. Borchman D, Foulks GN, Yappert MC, et al. Human meibum lipid conformation and thermodynamic changes with meibomian-gland dysfunction. Invest Ophthalmol Vis Sci. 2011;52(6):3805-3817. doi:10.1167/iovs.10-6514

20. Kenrick CJ, Alloo SS. The limitation of applying heat to the external lid surface: a case of recalcitrant meibomian gland dysfunction. Case Rep Ophthalmol. 2017;8(1):7-12. doi:10.1159/000455087

21. Lane SS, DuBiner HB, Epstein RJ, et al. A new system, the LipiFlow, for the treatment of meibomian gland dysfunction. Cornea. 2012;31 (4):396-404. doi:10.1097/ICO.0b013e318239aaea
22. Hura AS, Epitropoulos AT, Czyz CN, Rosenberg ED. Visible meibomian gland structure increases after vectored thermal pulsation treatment in dry eye disease patients with meibomian gland dysfunction. Clin Ophthalmol. 2020;14:4287-4296. doi:10.2147/OPTH.S282081

23. Goldberg DJ. Current trends in intense pulsed light. J Clin Aesthet Dermatol. 2012;5(6):45-53.

24. Sabeti S, Kheirkhah A, Yin J, Dana R. Management of meibomian gland dysfunction: a review. Surv Ophthalmol. 2020;65(2):205-217. doi:10.1016/j.survophthal.2019.08.007

25. Ma X, Lu Y. Efficacy of intraductal meibomian gland probing on tear function in patients with obstructive meibomian gland dysfunction. Cornea. 2016;35(6):725-730. doi:10.1097/ICO.0000000000000777

26. Butovich IA, Millar TJ, Ham BM. Understanding and analyzing meibomian lipids-a review. Curr Eye Res. 2008;33(5):405-420. doi:10.1080/02713680802018419

27. Sridharan K, Sivaramakrishnan G. Therapies for meibomian gland dysfunction: a systematic review and meta-analysis of randomized controlled trials. Open Ophthalmol J. 2017;11:346-354. doi:10.2174/ 1874364101711010346

28. Zhao Y, Veerappan A, Yeo S, et al. Clinical trial of thermal pulsation (LipiFlow) in meibomian gland dysfunction with preteatment meibography. Eye Contact Lens. 2016;42(6):339-346. doi:10.1097/ ICL.0000000000000228

29. Finis D, Hayajneh J, Konig C, et al. Evaluation of an automated thermodynamic treatment (LipiFlow(R)) system for meibomian gland dysfunction: a prospective, randomized, observer-masked trial. Ocul Surf. 2014;12(2):146-154. doi:10.1016/j.jtos.2013.12.001

30. Finis D, Konig C, Hayajneh J, et al. Six-month effects of a thermodynamic treatment for MGD and implications of meibomian gland atrophy. Cornea. 2014;33(12):1265-1270. doi:10.1097/ ICO.0000000000000273

31. Greiner JV. A single LipiFlow(R) Thermal Pulsation System treatment improves meibomian gland function and reduces dry eye symptoms for 9 months. Curr Eye Res. 2012;37(4):272-278. doi:10.3109/ 02713683.2011 .631721

32. Greiner JV. Long-term (3 year) effects of a single thermal pulsation system treatment on meibomian gland function and dry eye symptoms. Eye Contact Lens. 2016;42(2):99-107. doi:10.1097/ ICL.0000000000000166
Clinical Ophthalmology

\section{Publish your work in this journal}

Clinical Ophthalmology is an international, peer-reviewed journal covering all subspecialties within ophthalmology. Key topics include: Optometry; Visual science; Pharmacology and drug therapy in eye diseases; Basic Sciences; Primary and Secondary eye care; Patient Safety and Quality of Care Improvements. This journal is indexed on PubMed

\section{Dovepress}

Central and CAS, and is the official journal of The Society of Clinical Ophthalmology (SCO). The manuscript management system is completely online and includes a very quick and fair peer-review system, which is all easy to use. Visit http://www.dovepress.com/ testimonials.php to read real quotes from published authors. 